\title{
Research on Recommended and Comparative Strategy of Electronic Commerce
}

\author{
Hongtao Jiang ${ }^{1, a}$ \\ ${ }^{1}$ Chongqing Technology and Business Institute, Chongqing, 400052 \\ ${ }^{a}$ email
}

Keywords: Electronic Commerce, Recommended and Comparative Strategy

\begin{abstract}
The popularity of e-commerce and the rapid development of the Internet has changed people's traditional way of doing business. More and more people are willing to buy goods online, but also because of the convenience of e-commerce companies and a lot of profit. But e-commerce also contributed to the prosperity of information overload, a large number of users in the product information in a relatively short time is difficult to find the goods they need. E-commerce recommendation system came into being. It can directly interact with the user, to help users find the merchandise, so the successful completion of the purchase process. E-commerce recommendation system can effectively retain customers and prevent customer churn, improve marketing and e-commerce businesses competitive.
\end{abstract}

\section{Introduction}

In recent years, the rapid increase in number of Internet users, prompting frequent flow of online information, resulting in information overload (Information Overload) [3] [4]. More and more sites offer information for the user at the same time, the structure becomes more complex, how timely the mass of information on the Internet found the required information has become increasingly difficult. IEEE Internet Computing 2001 years, statistics show that the Internet produces 2x1018 terabytes of information every year, the famous search engine Google to search only $1.3 \times 108$ trillion pages. So excess message to the user a lot of inconvenience. On the one hand, users face a lot of information helpless, often lost in the almost unlimited information space, customers use more time to browse the product information they need and are compared to find the most suitable products. In fact, the customer is difficult to choose from an excess of information that they are most satisfied with the product information; on the other hand, the site has lost contact with the user, the business is also facing many new challenges, including product quality assurance, delivery timely product selection and comfortable, easy return, while constantly developing new industry opportunities, provide a lot of product information. One way to address these issues in e-commerce is to develop an intelligent recommendation system, providing customers with personalized product information services. Recommended system can help e-commerce sites effectively improve the attractiveness of products to enable enterprises to get as much benefit to help users in thousands of products, a better choice of goods to their own satisfaction, but also to solve the problem of information overload It provides a more effective method.

\section{Recommended System}

Recommended system has many applications. Before 1997, the recommended system is mainly used for filtering information and e-commerce, such as e-mail filtering, filter news articles, etc., on behalf of the system has GruopLens, PHOAKS, Fab, Referral Web, SiteSeer like. Since 1997, there has been a new recommendation system applications - e-commerce. After the introduction of recommender systems, e-commerce system can predict the user's preferences, the user may only be interested in forming a list of items recommended to the user, the customer can easily find products they are interested in, not only a great convenience to customers, and potentially increasing the volume of e-commerce. Personalized recommendation system makes the e-commerce system actively adapt to the specific needs of each user, for the user to create an electronics store for each 
user to provide different personalized shopping experience for each user, the e-commerce system to achieve " one to one marketing "personalized service possible. Thus, e-commerce businesses have begun to recommend to their e-commerce system embedded system, triggering a wave of recommender systems research and applications. The biggest advantage is that the recommendation system that can collect information on user interest and in accordance with user preferences for users to take the initiative to make personalized recommendations. Recommended composition of the entire system can be divided into three modules: an input module (the Input functional), the recommended method module (the Recommendation method), the output module (the Output functional). Among them, the recommended method module is the core part of the recommendation system, determines the performance of the merits of the recommendation system. Different types of recommendation system, the input information are also different. Enter the recommendation system may be the user's current behavior, the user can also access the history of the process behavior. In large recommendation system,

\section{Main Recommendation Technology}

Collaborative filtering (collaborative filtering recommendation) is currently the most studied personalized recommendation technology, which is based on the user's profile to get a neighbor recommended target user, recommended high degree of personalization. The famous system GroupLens / NetPerceptions, Ringo / Firefly and Tapestry and so on. The biggest advantage of collaborative filtering recommendation is subject no special requirements, can handle unstructured complex objects, such as music, movies.

Clustering refers to a set of physical or abstract objects grouped into a plurality of classes by a process similar objects. Generated by the clustering of class is a collection of objects, these objects with the same class of objects similar to each other, and other classes of objects distinct from each other. Standard clustering is similarity within the class as large as possible, the similarity between the classes as small as possible. Clustering can be roughly divided into two categories: the clustering of objects known as Q-type clustering, often used to measure the similarity distance or similarity coefficient; while the property is called clustering $\mathrm{R}$ cluster, it is usually based on the correlation coefficient or correlation coefficient to measure the similarity.

Content-based filtering recommendation system using information content (such as text documents) and the similarity of interest to filter information, they use similar resources and interest to filter information. Based on the recommendations, also known as information filtering based on recommendation by the information retrieval (Information Retrieve) areas proposed, thus the use of a number of IR skill in the art. Content-based recommendation basic idea is: for each user with a user's interests called file (User Profile) file into a data structure to describe their preferences, and the contents of each item of the feature extraction (Feature Extraction), formed feature vector (feature vector). When a user needs to be recommended to the user's file with user interest feature matrix comparing all items obtained similarity between the two, the system recommended by the similarity of the document. Some systems also collect user feedback information to facilitate maintenance user interest file. The key to this technique is that the document to be filtering feature extraction (that means the document), expressed interest in the file and the user similarity computing.

\section{Recommendations Strategy and Its Process}

Network development and popularization of changed people's way of life will gradually become an everyday essential. Network era, the individual needs of consumers of the performance is more prominent, such as requiring a variety of goods to choose from, or would like companies to produce customized products; access to information but also more proactive, this Internet age they are good at making psychological adventure and willing to carry out two-way communication, unilateral provision of information can not meet people's needs; consumer brand awareness has become more rational, with a certain brand preference; consumers will be shopping as a recreational one way, 
personalized services and products can be obtained favor. According to the Centre for Retail Research Internet Vanderbilt University in the US said, adding personalized content and personalized services to foster customer loyalty in the site. The system is a personalized recommendation based on your personal preferences, habits and transaction records to recommend the program to their information, goods, and it can be in a variety of ways to provide personalized information services. Different types of products, its application recommendation system, and suggested strategies employed are not the same. For different types, the use of appropriate policy recommendations in order to better network marketing, better achieve the effect of the recommendation system applications. Based on customer purchase frequency division type of product, you can put products into products and non-frequent purchases of frequently purchased products. From this type of analysis and research division to recommend strategies.

Frequent purchase of products, such as food, daily necessities, books, CD, DVD, etc., these low commodity prices and the wide variety of goods. And the user will frequently make a purchase in a short time, and therefore the site has a lot to keep customers' personal information and purchase history information. For these products, it is recommended by user rating system for different commodities adjacent to obtain recommendation result, or by analyzing the customer's personal information, browsing history, and previous online purchase history inferred his personal parameters. Once the customer's personal information, the recommendation system can create a computational model to predict the customer for the customer in the same fields of application he might prefer another product. Therefore, this type of recommendation system is the core of customers' personal learning parameters and ability to adapt to changes in customer interest, and accurate modeling of the customer's preferences. It can gather information of interest to the user, based on user interest and user preferences initiative to make personalized recommendations. Thus, every time a user name and password to log e-commerce site, the system will automatically recommend recommended to the user's favorite $\mathrm{N}$ products according to user preference level, and recommendation given is updated in real time. That is, when the system of portfolio and interest-profile changes, the recommended sequence given automatically changed greatly facilitates the user to browse information on the goods, but also improve the company's level of service.

\section{Establishment of Product Recommendation Process}

Customer demand is collected from the interface. Assume that most customers do not know the relevant domain knowledge, so the problem at the interface can not be quantified or very specialized, user-friendly to use more qualitative issues. As can be determined roughly customer requirements for products from the customer's use of the occasion and some clear indicators of their requirements or preferences. After collecting the information to the customer's needs, fuzzy evaluation agreed to represent the client's needs. In describing the product, first of all determined by experts in various product indicators and their weights and technical parameters, and using expert scoring method to determine the respective weight Analytic Hierarchy Process. An important indicator of the product by its corresponding technical parameters after the addition of the weight factor with the agreed rules and fuzzy evaluation, ie, product characterization. Then according to the weight of index, calculated the comprehensive capacity of the product. Since then, customer demand for the product description and comprehensive ability similarity comparison, representatives from the small big similarity. Recommended result in the form of this article taken to top- $\mathrm{N}$, it is given in the calculation of the similarity of the largest $\mathrm{N}$ products.

If the customer needs and product comparison database of all products, although the products are all products can be recommended as candidates, but doing so is a waste of time and inefficiency, and unnecessary. For example, if the customer wants to buy the product price is relatively high in the same kinds of products, it is not necessary for the lower recommended price of the product, so no need to consider before calculating the lower-priced product, and do not be similarity computing customers demand. Again, if the customer has its special brand preferences, you can simply recommend products under the brand, and other brands Recommended for unnecessary products, so there is no need to consider other branded products to the customer needs similar degree. Therefore, 
in this paper, mainly through price and brand of these two indicators, the first product and customer demand for simple classification. After classification, only with product and customer needs within the category as compared to the case since it greatly reduces the amount of calculation and improve efficiency, particularly in commodities continues to grow rapidly, the database is extremely complex and bulky case. Price indicators give a clear definition of the interface, customers can choose their own can bear the price of the prompts. Customer demand for the same price and product similarity calculation, only for customers recommend him to withstand the price of the product. Taking into account the possibility of a customer's product brand has a special preference, Brand added. When a customer interface section explain their brand when demand is high, we assume there is high demand for the brand who are loyal to a particular brand advocates, the next step will be allowed to choose their preferred brand.

\section{Conclusion}

In the context of lifelong learning society, to provide personalized services digital learning platform is essential for life-long learning system. E-Learning as a lifelong learning system, the most important way of learning, its personalized construction has important practical significance. In this study, the Ministry of Education Humanities and social sciences research project "Personal for lifelong learning digital learning service model research" sub-project, to propose recommended strategy E-Learning personalized recommendation system, improve E-Learning in personalized recommendation Theory to meet the needs of the development of lifelong learning digital learning platform foundation.

\section{References}

[1] Abbattista F, Degemmis M, Licchelli O, Lops P, Semeraro G and Zambetta F. Improving the Usability of an E-Commerce Web Site through Personalization. In: Proceedings of the Workshop on Recommendation and Personalization in Ecommerce, 2002.

[2] Salton G and Buckley C. Improving Retrieval Performance by Relevance Feedback. Journalof the American Society for Information Science, 1990.

[3] Sarwar B, Karypis G, Konstan J and Reid1 J. Item-based Collaborative Filtering Recommen-lotion Algorithms. In Proceedings of the Tenth International World Wide Web Conference on World Wide Web. 2001.

[4] Wofl J, Aggarwal C, Wu K-L and Yu P. Horting Hatches an Egg : A New Graph-theoreticApproach to Collaborative Filtering. In: Proceedings of ACM SIGMOD International Conference on Knowledge Discovery \& Data Mining, 2009. 